\title{
Case Report: Rifampicin-Induced Thrombocytopenia in a Patient with Borderline Lepromatous Leprosy
}

\author{
Tiejun Shui, ${ }^{1} \dagger$ Chao Shi, ${ }^{2} \dagger$ Zhichun Jing, ${ }^{2}$ Degang Yang, ${ }^{2}$ and Jianyu Zhu ${ }^{2 *}$ \\ ${ }^{1}$ Department of Leprosy Control, Yunnan Center for Disease Control and Prevention, Kunming, People's Republic of China; ${ }^{2}$ Department of \\ Leprosy, Shanghai Dermatology Hospital, Shanghai, People's Republic of China
}

\begin{abstract}
Rifampicin is a highly effective antibacterial drug and an important component of multidrug therapy used to treat leprosy. Side effects of rifampicin are rare with the once-a-month dosage regimen of anti-leprosy multidrug therapy. Here, we report a case of rifampicin-induced thrombocytopenia during anti-leprosy treatment. Although rare, this potential side effect merits attention.
\end{abstract}

\section{INTRODUCTION}

Leprosy is a chronic contagious disease caused by the Mycobacterium leprae. It mainly affects the skin and peripheral nerves and may cause permanent nerve damage and disability. Leprosy is still endemic in some regions of the world. According to the WHO records, more than 200,000 new leprosy cases are reported every year. ${ }^{1,2}$

In China, patients with leprosy are treated with the multidrug therapy regimen recommended by the WHO, composed of dapsone, rifampicin, and clofazimine. Although well tolerated, rifampicin still has some side effects, some of which can lead to serious consequences. ${ }^{2,3}$ Here, we report a case of thrombocytopenia caused by the side effects of rifampicin in a leprosy patient during anti-leprosy treatment.

\section{CASE PRESENTATION}

A 75-year-old Chinese man came to our hospital in 2018 with a 2-year history of multiple paresthetic erythematous plaques over his face and limbs. The patient also had pronounced bilateral tender ulnar nerve and common peroneal nerve thickening. Slit-skin smear examinations were positive for acid-fast bacilli (bacterial index 2). Histologic examination of an erythematous plaque on the right upper limb showed a clear zone beneath the epidermis separating macrophage granulomata in the dermis from the atrophic epidermis, which is consistent with the histopathologic characteristics of borderline lepromatous leprosy. He was diagnosed with borderline lepromatous leprosy.

Anti-leprosy treatment was initiated with multidrug therapy (600 mg of rifampin and $300 \mathrm{mg}$ of clofazimine monthly; $100 \mathrm{mg}$ of dapsone and $50 \mathrm{mg}$ of clofazimine daily). Five days later, he complained of skin plaques. Physical examination indicated palpable, non-blanching purpura on the skin of both upper and lower limbs. Several small ulcers were noted on his oral mucosa. There was no hepatosplenomegaly or lymphadenopathy. Laboratory examinations showed a platelet count of $18 \times 10^{9} / \mathrm{L}\left(166 \times 10^{9} / \mathrm{L}\right.$ recorded 5 days before the onset of these new symptoms) and a hemoglobin concentration of $10.9 \mathrm{~g} / \mathrm{dL}$. White cell count and erythrocyte count were normal. Liver and renal function tests were normal.

\footnotetext{
*Address correspondence to Jianyu Zhu, Shanghai Dermatology Hospital, No. 1278 Bao De Rd., Shanghai 200443, People's Republic of China. E-mail: 35476451@qq.com

†These authors contributed equally to this work.
}

Serum concentrations of autoantibodies, complement, and immunoglobulin were normal. Viral markers of hepatitis $B$ and $C$, and acquired immunodeficiency syndrome were negative. Prothrombin time was 11.6 seconds (normal 10-14 seconds), activated partial thromboplastin time was 25.6 seconds (normal 26-36 seconds), thrombin time was 15.8 seconds (normal 12-16 seconds), and fibrinogen concentration was $3.48 \mathrm{~g} / \mathrm{L}$ (normal 2-4 g/L). Examination of a bone marrow smear showed bone marrow proliferated actively, and there was a pronounced increase in megakaryocytes. According to his medical history and clinical symptoms, we confirmed the diagnosis of borderline lepromatous leprosy with concurrent thrombocytopenia.

Anti-leprosy treatment was stopped. During the next 48 hours, the patient was transfused 16 units of platelet concentrates. Five days later, his platelet count increased to $145 \times$ $10^{9} / \mathrm{L}$ and clinical symptoms improved. One month later, antileprosy multidrug therapy was restarted with rifampicin, dapsone, and clofazimine after taking full precautions and acquiring informed consent from the patient. By the fifth day, his platelet count decreased to $20 \times 10^{9} / \mathrm{L}$, and he developed purpura and bleeding gums. We gave the patient a transfusion of concentrated platelets and continued to treat his leprosy with clofazimine and dapsone. After 5 days, his platelet count was back to normal.

The patient refused to be treated with second-line antileprosy drugs recommended by the WHO for fear of recurring thrombocytopenia. In the subsequent anti-leprosy treatment, only clofazimine and dapsone were given to the patient, and rifampicin was never used again. The patient did not suffer from thrombocytopenia again. This evidence suggested that thrombocytopenia observed in the patient was most likely induced by rifampicin.

The patient was followed up in our outpatient department and treated with clofazimine and dapsone for leprosy for 12 months. His hematological parameters were monitored and remained normal.

\section{DISCUSSION}

The WHO has recommended multidrug therapy for leprosy in 1981. The WHO currently recommends 12 months of treatment with a combination of $600 \mathrm{mg}$ of rifampin once per month, $300 \mathrm{mg}$ of clofazimine once per month, and daily doses of clofazimine $(50 \mathrm{mg})$ and dapsone $(100 \mathrm{mg})$ to treat leprosy. ${ }^{2,3}$ Rifampicin inhibits DNA-dependent RNA polymerase in bacterial cells by binding its beta-subunit, thereby 
preventing transcription to RNA and subsequent translation to proteins. ${ }^{4,5}$ Gastrointestinal problems (nausea, vomiting, diarrhea, and abdominal pain), hepatotoxicity, drug eruption, flu syndrome, hemolytic anemia, and renal failure are common side effects of rifampicin, particularly during intermittent treatment, but thrombocytopenia is rare. ${ }^{3,5,6}$

In this report, the patient received anti-leprosy treatment including rifampicin for the first two rounds. Both times, the patient developed thrombocytopenia. The platelet count reached normal levels several days after discontinuing rifampicin treatment. In subsequent anti-leprosy treatment, patients were not given rifampicin, and no further thrombocytopenia occurred. For this reason, we speculated that thrombocytopenia was induced with rifampicin. Most of the drugs induce thrombocytopenia by immunologic platelet destruction. Pereira et al. ${ }^{7}$ reported that the glycoprotein lb/ IX complex is the target in rifampicin-induced immune thrombocytopenia. If patients develop thrombocytopenia, rifampicin should be discontinued immediately and should not be used in the future. . $^{3,5}$

In the process of diagnosis, we need to evaluate the differential diagnosis between thrombocytopenic purpura induced with rifampicin and purpura dermatitis caused by dapsone hypersensitivity syndrome (DHS). Dapsone hypersensitivity syndrome is a rare but well-recognized serious adverse effect characterized by fever, skin eruption, generalized lymphadenopathy, and liver abnormalities (hepatomegaly, hepatitis, jaundice, deranged liver function tests, or some combination of these). The interval between the start of dapsone therapy and appearance of DHS symptoms varied from 2 to 8 weeks. ${ }^{8,9}$ Dapsone hypersensitivity syndrome may occur in patients with leprosy who receive anti-leprosy multidrug therapy containing dapsone. The patient's clinical manifestations and laboratory examinations did not correspond with the diagnostic criteria of DHS. Although rifampicin-induced thrombocytopenia is rare during anti-leprosy treatment, it should be handled promptly to avoid serious consequences.

Received March 23, 2020. Accepted for publication June 26, 2020.

Published online August 3, 2020.

Acknowledgment: We thank LetPub (www.letpub.com) for its linguistic assistance during the preparation of this manuscript.

Authors' addresses: Tiejun Shui, Department of Leprosy Control, Yunnan Center for Disease Control and Prevention, Kunming, People's Republic of China, E-mail: 67637539@qq.com. Chao Shi, Zhichun Jing, Degang Yang, and Jianyu Zhu, Department of Leprosy, Shanghai Dermatology Hospital, Shanghai, People's Republic of China, E-mails: sc_mac@163.com, 743835016@qq.com, ydg007@ gmail.com, and 35476451@qq.com.

\section{REFERENCES}

1. World Health Organization, 2019. Global leprosy update, 2018: moving towards a leprosyfree world. Wkly Epidemiol Rec 94: 389-411.

2. Kar HK, Gupta R, 2015. Treatment of leprosy. Clin Dermatol 33: 55-65.

3. Noordeen S, 2016. History of chemotherapy of leprosy. Clin Dermatol 34: 32-36.

4. Bera S, Mondal D, 2019. Insights of synthetic analogues of antileprosy agents. Bioorg Med Chem 27: 2689-2717.

5. Bullock WE, 1983. Bullock. Rifampicin in the treatment of leprosy. Rev Infect Dis 5 (Supp/ 3): S606-S613.

6. Agrawal A, Gutch M, Jain N, Singh A, 2012. Do not miss rifampicininduced thrombocytopenic purpura. BMJ Case Rep 2012: bcr1220115282.

7. Pereira J, Hidalgo $P$, Ocqueteau M, Blacutt M, Marchesse M, Nien Y, Letelier L, Mezzano D, 2000. Glycoprotein Ib/IX complex is the target in rifampicin-induced immune thrombocytopenia. $\mathrm{Br}$ $J$ Haematol 110: 907-910.

8. Wang N, Parimi L, Liu H, Zhang F, 2017. A review on dapsone hypersensitivity syndrome among Chinese patients with an emphasis on preventing adverse drug reactions with genetic testing. Am J Trop Med Hyg 96: 1014-1018.

9. Agrawal S, Agarwalla A, 2005. Dapsone hypersensitivity syndrome: a clinico-epidemiological review. J Dermatol 32: 883-889. 